\title{
Prevalence and risk factors associated with HIV and syphilis co-infection in the African Cohort Study: a cross-sectional study
}

\author{
Laura Gilbert ${ }^{1 *}$, Nicole Dear ${ }^{1,2}$, Allahna Esber ${ }^{1,2}$, Michael Iroezindu ${ }^{1,3}$, Emmanuel Bahemana ${ }^{1,4}$, \\ Hannah Kibuuka ${ }^{5}$, John Owuoth ${ }^{6,7}$, Jonah Maswai ${ }^{1,8}$, Trevor A. Crowell ${ }^{1,2}$, Christina S. Polyak ${ }^{1,2}$ and \\ Julie A. Ake ${ }^{1}$ on behalf of the AFRICOS Study Group
}

\begin{abstract}
Background: Each year, 5.6 million new syphilis cases are diagnosed globally. Guidelines for people living with HIV $(P L W H)$ in low-income countries (LIC) recommend STI testing for symptomatic persons and those newly diagnosed with HIV; routine STI testing is less clear. Here we provide updated syphilis prevalence and identify co-infection risk factors in PLWH in the African Cohort Study (AFRICOS) to understand these rates as they relate to syndromic treatment.

Methods: AFRICOS is a study enrolling PLWH and HIV-uninfected individuals in four African countries. Participant study enrollment information was used to determine syphilis prevalence and co-infection risk factors. Inclusion criteria consisted of adults 18 years or older receiving care at a participating clinic as a long-term resident who consented to data and specimen collection. Exclusion criteria consisted of pregnancy and/or imprisonment. Screen-positive syphilis was defined as a reactive rapid plasma regain (RPR) upon study enrollment whereas confirmed syphilis included a reactive RPR followed by reactive treponemal test. Multivariate analyses was performed to determine HIV and syphilis co-infection risk factors.

Results: Between 2013 and March 1, 2020, 2939 PLWH enrolled and 2818 were included for analysis. Screen-positive and confirmed syphilis prevalence were 5.3\% (151/2818) and 3.1\% (87/2818), respectively. When the analysis was restricted to PLWH with an RPR titer of greater than, or equal to, 1:8, 11/87 (12.6\%) participants were included. No PLWH and confirmed syphilis had documented genital ulcers. In the multivariate model, participants with confirmed syphilis co-infection were more likely to have none or some primary education [aOR $3.29(1.60,6.74)]$ and consume alcohol [aOR $1.87(1.16,3.03)]$ compared to those without syphilis. Antiretroviral therapy (ART) with suppressed viral load $(\mathrm{VL})$ was protective in the unadjusted model but not adjusted multivariate model.

Conclusions: Our findings show that syphilis rates in sub-Saharan Africa remain elevated where diagnosis remains challenging, and that both lower education level and alcohol consumption are significantly associated with HIV/syphilis co-infection in AFRICOS. Based on our analysis, current STI guidelines targeting testing for African individuals with either new HIV diagnosis or syndromic symptoms may be inadequate, highlighting the need for increased testing and treatment strategies in resource-limited settings.
\end{abstract}

\footnotetext{
*Correspondence: laura.j.gilbert.mil@mail.mil

1 U.S. Military HIV Research Program, Walter Reed Army Institute

of Research, Silver Spring, MD, USA
}

Full list of author information is available at the end of the article

(C) The Author(s) 2021. Open Access This article is licensed under a Creative Commons Attribution 4.0 International License, which permits use, sharing, adaptation, distribution and reproduction in any medium or format, as long as you give appropriate credit to the original author(s) and the source, provide a link to the Creative Commons licence, and indicate if changes were made. The images or other third party material in this article are included in the article's Creative Commons licence, unless indicated otherwise in a credit line to the material. If material is not included in the article's Creative Commons licence and your intended use is not permitted by statutory regulation or exceeds the permitted use, you will need to obtain permission directly from the copyright holder. To view a copy of this licence, visit http://creativecommons.org/licenses/by/4.0/. The Creative Commons Public Domain Dedication waiver (http://creativeco mmons.org/publicdomain/zero/1.0/) applies to the data made available in this article, unless otherwise stated in a credit line to the data. 
Keywords: Human immunodeficiency virus (HIV), Syphilis, Africa

\section{Background}

Every year, 5.6 million new syphilis cases are diagnosed in men and women ages $15-49$ years old globally $[1,2]$. Comprehensive studies on syphilis prevalence in low-income countries (LIC) are mostly extrapolated from antenatal care clinics where prevalence is estimated at $3.5-4.6 \%$ in Africa (excluding South Africa) [2, 3]. In PLWH, a population where co-infections with sexually transmitted infections (STIs) frequently coexist due to their shared mode of sexual transmission, few studies have researched syphilis prevalence in LIC through examination of serologic confirmatory testing, largely due to diagnostic challenges. Capturing an accurate assessment of syphilis prevalence in PLWH is of particular public health concern because it not only increases the likelihood of HIV transmission, but also alters the natural history of HIV, where PLWH experience a temporary reduction in CD4 counts and elevation in HIV viral load (VL) in early, untreated syphilis infections [4]. Besides the epidemiological aspect, the co-existence of these two infectious diseases is also of clinical and diagnostic interest: PLWH more commonly demonstrate atypical clinical features of syphilis and serological interpretation of syphilis testing may not correlate with clinical presentation in this population [5].

Current World Health Organization (WHO) guidelines for PLWH living in resource-limited settings (published in 2008) recommend STI testing only for symptomatic individuals as well as those with a new HIV diagnosis, possibly leading to a large proportion of undiagnosed STIs and continued community spread [6, 7]. For treatment of syphilis, the WHO recommends intramuscular benzathine penicillin (one dose in early syphilis and once weekly for three consecutive weeks in late or unknown syphilis) [8]. Routine STI testing is less clear and thus, updated data on HIV and syphilis co-infection prevalence and risk factors is urgently needed in order to not only understand these rates as they are related to using syndromic treatment but also to guide newer recommendations for PLWH, especially if Africa is to attain the goal of $90 \%$ reduction of syphilis by 2030 as outlined by the WHO [2]. Here we provide epidemiologic information and describe significant risk factors for PLWH and serologically confirmed syphilis co-infection in the African Cohort Study (AFRICOS).

\section{Methods}

\section{Description of AFRICOS Cohort}

AFRICOS is an ongoing longitudinal study enrolling PLWH and HIV-uninfected individuals at 12 large public and private health facilities supported through the
US Military HIV Research Program (MHRP) by the President's Emergency Plan for AIDS Relief (PEPFAR) in Kenya, Tanzania, Uganda, and Nigeria. Its objective is to assess the impact of clinical practices, biological factors, and socio-behavioral issues on HIV infection and disease progression in African settings with aims of informing practice and policy [9]. Enrollment began in January 2013. Individuals were eligible if they were 18 years or older, receiving care at an enrolling PEPFAR clinic, intended to be a long-term resident of the area, willing to provide demographic information and be contacted by the study staff, and consented to data and specimen collection. Exclusion criteria consisted of any significant condition that in the opinion of the study investigator might interfere with the conduct of the study, pregnancy, or if the subject was a prisoner [9]. All participants provided written informed consent. The study was approved by institutional review boards of the Walter Reed Army Institute of Research, Makerere University School of Public Health, Kenya Medical Research Institute, Tanzania National Institute of Medical Research, and the Nigerian Ministry of Defense [9].

Demographic, laboratory (including HIV-specific and syphilis testing) as well as clinical information (including physical exam) is captured upon AFRICOS enrollment and at each bi-annual visit for every AFRICOS participant. For this cross-sectional study, participant information gathered at time of AFRICOS enrollment was used to determine syphilis screen-positive and confirmed syphilis prevalence, as well as risk factors for HIV and syphilis co-infection. Because untreated syphilis is associated with long-term neurologic sequelae, cognitive impairment is also assessed in AFRICOS participants. Measurement of cognitive impairment upon AFRICOS enrollment was with the International HIV Dementia Scale (IHDS), which includes evaluation of memory recall, motor speed and psychomotor speed [10].

\section{Study enrollment questionnaire}

Risk factors for HIV and syphilis co-infection were ascertained through examination of study participants' responses to a comprehensive behavioral questionnaire upon AFRICOS enrollment. The questionnaire included information regarding demographics (including education history), sexual history and behaviors, social history and behaviors (including substance abuse), general health and medical conditions, as well as cognition. Education was categorically defined by participants selecting their highest level of education completed. Alcohol 
consumption was dichotomously defined as either "Yes" or "No" in response to the question: "Do you consume alcohol?".

\section{Syphilis diagnosis}

All countries utilize the rapid plasma reagin (RPR) as the initial (non-treponemal) screening test followed by a confirmatory (treponemal) test, where most countries use the Treponema pallidum antibody (TPA) as the treponemal test. Screen-positive syphilis was defined as having only a reactive RPR upon study enrollment, where any RPR titer that was greater than, or equal to, 1:2 was included in the analysis. Serologically-confirmed syphilis was defined as having a reactive RPR as well as a reactive treponemal test upon study enrollment. AFRICOS participants with a diagnosis of serologically-confirmed syphilis and a reactive RPR titer equal to, or greater than, 1:8 were further analyzed given their higher likelihood of having an active syphilis infection. All countries in this study utilized the traditional syphilis diagnostic algorithm (no countries utilized the reverse syphilis algorithm). Syphilis diagnostic testing was performed in an AFRICOS-associated laboratory and no point of care (POC) syphilis testing was included in this analysis.

\section{Statistical design}

Bivariate analyses using $\chi^{2}$ tests for categorical variables were conducted to compare cases of confirmed syphilis by demographic, clinical, and socio-behavioral factors. All the independent variables investigated in our analyses had less than $6 \%$ missing data. Logistic regression models were used to estimate unadjusted and adjusted odds ratios and 95\% confidence intervals (95\% CIs) for associations between various factors and confirmed syphilis. Factors with a p-value $<0.05$ from the bivariate analysis were included in the multivariate model along with additional factors identified a priori as being relevant to syphilis. Analyses were performed in SAS version 9.3 (SAS Institute, Cary, North Carolina) and Stata version 15.0 (StataCorp, College Station, Texas) software.

\section{Results}

\section{Characteristics of all AFRICOS participants}

Between January 21, 2013 and March 1, 2020, 2939 PLWH were enrolled into AFRICOS and 2818 had complete covariate and outcome data and were included in this analysis. A total of 1649/2818 (58.5\%) of participants were female and median age was 38.3 (Interquartile range, IQR: 31,46$)$ years old. The highest enrollment site was South Rift Valley, Kenya (36\% of participants) and most frequent education category was primary or some secondary (39\% of participants). A total of 1986/2818 (70\%) participants were taking antiretroviral therapy
(ART) upon study enrollment where the median time since HIV diagnosis was 2.5 (IQR: $0.2-6.3$ ) years and the median time on ART was 3.0 (IQR: 0.7-5.9) years. Median CD4 count for all PLWH enrolled was 390 (IQR: 238,573$)$ cells $/ \mathrm{mm}^{3}$ and $1361 / 2818(48.3 \%)$ had a HIV viral load less than 50 copies $/ \mathrm{mL}$ while taking ART (Table 1).

\section{Prevalence of screen-positive and serologically confirmed syphilis}

Prevalence of screen-positive and confirmed syphilis upon AFRICOS enrollment in PLWH was 5.3\% $(151 / 2818)$ and $3.1 \%(872818)$, respectively. None of the PLWH and confirmed syphilis co-infection had documented genital ulcers on enrollment exam (prevalence of $1.5 \%$ among PLWH overall). When the analysis was restricted to PLWH who had an RPR titer of greater than, or equal to, 1:8, 11/87 (12.6\%) participants were included. Further comparison of PLWH with confirmed syphilis among those with high and low titers was not able to be performed due to the small sample size resulting in insufficient power for analysis.

\section{Characteristics of PLWH and serologically confirmed syphilis}

Bivariate analysis for risk factors associated with HIV and serologically confirmed syphilis co-infection was performed on enrollment data for all PLWH (Table 1). Among PLWH with serologically confirmed syphilis $(\mathrm{n}=87)$, a total of $45 / 87(51.7 \%)$ were female, median age was $38(32,45)$ years and $56.2 \%$ were married. Additionally, PLWH and confirmed syphilis had lower median CD4 counts ( $p$-value $<0.01$ ) and fewer were virally suppressed compared to those without syphilis $(39.1 \%$ vs $48.6 \%$, respectively) (p-value $<0.001$ ). They also had a shorter duration of both HIV diagnosis and ART administration.

\section{Multivariate analysis}

Multivariate analysis was performed to assess risk factors for PLWH and syphilis co-infection and are displayed in Table 2 . In the multivariate model, statistically significant risk factors that were associated with an increased risk for HIV and syphilis co-infection included education level and alcohol consumption. Having no/some primary education (compared to PLWH and history of secondary education and above) increased the risk for HIV and syphilis co-infection [OR: 3.29 ; 95\% CI $(1.60,6.74)]$. Alcohol consumption also increased the risk for HIV and syphilis co-infection [OR: 1.87; 95\% CI $(1.16,3.03)]$. Analysis demonstrated that ART administration with VL less than 200 copies $/ \mathrm{mL}$ was considered protective in the 
Table 1 Baseline characteristics of PLWH and syphilis co-infection

\begin{tabular}{|c|c|c|c|c|}
\hline & All $(n=2818)$ & No syphilis $(n=2731)$ & Syphilis $(\mathrm{n}=\mathbf{8 7})$ & $P$ value \\
\hline Gender & & & & 0.19 \\
\hline Male & $1169(41.5 \%)$ & $1127(41.3 \%)$ & $42(48.3 \%)$ & \\
\hline Female & $1649(58.5 \%)$ & $1604(58.7 \%)$ & $45(51.7 \%)$ & \\
\hline Study site & & & & $<0.001$ \\
\hline Kayunga, Uganda & $515(18.3 \%)$ & $496(18.2 \%)$ & $19(21.8 \%)$ & \\
\hline South Rift Valley, Kenya & 1007 (35.7\%) & $977(35.8 \%)$ & $30(34.5 \%)$ & \\
\hline Kisumu West, Kenya & $499(17.7 \%)$ & $499(18.3 \%)$ & 0 & \\
\hline Mbeya, Tanzania & $495(17.6 \%)$ & $457(16.7 \%)$ & 38 (43.7\%) & \\
\hline Abuja \& Lagos Nigeria & $302(10.7 \%)$ & $302(11.1 \%)$ & 0 & \\
\hline Age at visit & & & & 0.13 \\
\hline $18-29$ & $506(18.0 \%)$ & $496(18.2 \%)$ & $10(11.5 \%)$ & \\
\hline $30-39$ & $982(34.8 \%)$ & $947(34.7 \%)$ & $35(40.2 \%)$ & \\
\hline $40-49$ & $819(29.1 \%)$ & $788(28.9 \%)$ & $31(35.6 \%)$ & \\
\hline $50+$ & $511(18.1 \%)$ & $500(18.3 \%)$ & $11(12.6 \%)$ & \\
\hline Age at visit ${ }^{\mathrm{a}}$ & $38.3(31.2,46.0)$ & $38.3(31.2,46.0)$ & $38.4(31.7,45)$ & 0.92 \\
\hline CD4 count (cells/uL) ${ }^{\mathrm{a}}$ & $390(238,573)$ & $393(239,575)$ & $323(182,456)$ & 0.006 \\
\hline CD4 count (cells/uL) & & & & 0.037 \\
\hline Less than 200 & $549(19.5 \%)$ & $526(19.3 \%)$ & $23(26.4 \%)$ & \\
\hline $200-499$ & $1313(46.6 \%)$ & $1268(46.4 \%)$ & $45(51.7 \%)$ & \\
\hline Greater than/equal to 500 & $956(33.9 \%)$ & $937(34.3 \%)$ & $19(21.8 \%)$ & \\
\hline \multicolumn{5}{|l|}{ Viral load (copies/mL) } \\
\hline Less than 50 & $1361(48.3 \%)$ & $1327(48.6 \%)$ & $34(39.1 \%)$ & $<0.001$ \\
\hline Greater than 50 & $588(20.9 \%)$ & $574(21 \%)$ & $14(16.1 \%)$ & \\
\hline Not on ART & $869(30.8 \%)$ & $830(30.4 \%)$ & 39 (44.8\%) & \\
\hline Education category & & & & 0.002 \\
\hline None/some primary & $947(33.6 \%)$ & $908(33.2 \%)$ & $39(44.8 \%)$ & \\
\hline Primary/some secondary & $1099(39.0 \%)$ & 1061 (38.9\%) & $38(43.7 \%)$ & \\
\hline Secondary and above & $772(27.4 \%)$ & $762(27.9 \%)$ & $10(11.5 \%)$ & \\
\hline Cognitive impairment ${ }^{b}$ & & & & 0.66 \\
\hline No & $2658(94.3 \%)$ & 2575 (94.3\%) & $83(95.4 \%)$ & \\
\hline Yes & $160(5.7 \%)$ & $156(5.7 \%)$ & $4(4.6 \%)$ & \\
\hline Consume alcohol & & & & 0.002 \\
\hline No & $2277(80.8 \%)$ & $2218(81.2 \%)$ & $59(67.8 \%)$ & \\
\hline Yes & $541(19.2 \%)$ & $513(18.8 \%)$ & $28(32.2 \%)$ & \\
\hline Support group & & & & 0.054 \\
\hline No & $2467(87.5 \%)$ & $2385(87.3 \%)$ & $82(94.3 \%)$ & \\
\hline Yes & $351(12.5 \%)$ & $346(12.7 \%)$ & $5(5.7 \%)$ & \\
\hline History of circumcision & & & & 0.051 \\
\hline Not circumcised & $396(14.1 \%)$ & $376(13.8 \%)$ & $20(23.0 \%)$ & \\
\hline Circumcised & $773(27.4 \%)$ & $751(27.5 \%)$ & $22(25.3 \%)$ & \\
\hline
\end{tabular}

${ }^{\mathrm{a}}$ Median, IQR; ${ }^{\mathrm{b}}$ International HIV Dementia Scale (IHDS)

unadjusted model but did not reach significance in the adjusted model.

\section{Discussion}

In this well-characterized cohort of PLWH in Africa, prevalence of screen-positive and confirmed syphilis coinfection was $5.3 \%$ and $3.1 \%$, respectively, demonstrating that syphilis rates remain elevated in the African context. Our results also demonstrate the challenges in the diagnosis of syphilis infection-and suggest a role for serologic confirmatory testing, as almost half of PLWH initially diagnosed with syphilis would have received unnecessary treatment based on results of the initial (non-treponemal) screening syphilis test alone. There are 
Table 2 Factors associated with syphilis co-infection among PLWH

\begin{tabular}{|c|c|c|}
\hline & $\begin{array}{l}\text { Unadjusted odds } \\
\text { ratio }(95 \% \mathrm{Cl})\end{array}$ & $\begin{array}{l}\text { Adjusted } \\
\text { odds ratio } \\
(95 \% \mathrm{Cl})\end{array}$ \\
\hline \multicolumn{3}{|l|}{ Gender } \\
\hline Female & $0.75(0.49,1.15)$ & \\
\hline \multicolumn{3}{|l|}{ Age (Years) } \\
\hline $18-29$ & Reference & Reference \\
\hline $30-39$ & $1.83(0.90,3.73)$ & $1.74(0.85,3.57)$ \\
\hline $40-49$ & $1.95(0.95,4.02)$ & $2.06(0.98,4.33)$ \\
\hline $50+$ & $1.09(0.46,2.59)$ & $1.19(0.49,2.93)$ \\
\hline \multicolumn{3}{|l|}{ Education level } \\
\hline None/some primary & $3.27(1.62,6.60)$ & $3.29(1.60,6.74)$ \\
\hline Primary/some secondary & $2.73(1.35,5.51)$ & $2.63(1.29,5.37)$ \\
\hline Secondary and above & Reference & Reference \\
\hline \multicolumn{3}{|l|}{ Cognitive impairment ${ }^{\mathrm{a}}$} \\
\hline Yes & $0.80(0.29,2.20)$ & $0.70(0.25,1.96)$ \\
\hline \multicolumn{3}{|l|}{ Alcohol consumption } \\
\hline Yes & $2.05(1.30,3.25)$ & $1.87(1.16,3.03)$ \\
\hline \multicolumn{3}{|l|}{ Support group involvement } \\
\hline No & $2.38(0.96,5.91)$ & $1.89(0.75,4.79)$ \\
\hline \multicolumn{3}{|l|}{ History of circumcision } \\
\hline Not circumcised & $1.90(0.11,3.25)$ & $1.44(0.82,2.51)$ \\
\hline Circumcised & Reference & Reference \\
\hline \multicolumn{3}{|l|}{ Viral suppression } \\
\hline On ART, VL <200 copies/mL & $0.50(0.32,0.80)$ & $0.65(0.39,1.08)$ \\
\hline On ART, VL > 200 copies $/ \mathrm{mL}$ & $0.66(0.34,1.24)$ & $0.73(0.38,1.40)$ \\
\hline Not on ART & Reference & Reference \\
\hline \multicolumn{3}{|l|}{ CD4 count $^{b}$} \\
\hline Less than 200 & $0.81(0.49,1.36)$ & $0.89(0.52,2.51)$ \\
\hline $200-499$ & $0.46(0.25,0.86)$ & $0.53(0.28,1.02)$ \\
\hline Greater than/equal to 500 & Reference & Reference \\
\hline
\end{tabular}

${ }^{a}$ International HIV Dementia Scale (IHDS); ${ }^{\text {b }}$ cells/uL

many reasons for a false-positive non-treponemal test, to include a diagnosis of HIV, and these findings highlight the need for increased testing capability in resource-limited settings.

The cohort's prevalence rates are slightly higher than prior African studies examining serologically confirmed syphilis: Djomand et al. reported an HIV and serologically confirmed syphilis co-infection rate of $0.6 \%$ in females and $2.4 \%$ in 559 newly diagnosed individuals with HIV entering care in their cross-sectional study Namibia [11]. In this study, the median age was 30 years (compared to 38 years in our study participants) and none were taking ART. The higher syphilis prevalence in our study may be associated with increased years of sexual activity and therefore longer duration of potential syphilis exposure in our cohort population. In a separate study, Singa et al. reported an overall rate of $0.5 \%$ in 1661
PLWH in Kenya [12]. This lower rate may be impacted by limited sexual risk behavior: most participants were married or cohabitating, and they reported high levels of condom use.

Notably, while prevalence of confirmed syphilis was $3.1 \%$ among PLWH, none of these participants had documented genital ulcers on exam. Only $12.6 \%(11 / 87)$ of the PLWH with syphilis co-infection in our study had an RPR titer of greater than, or equal to, 1:8 (which represents a higher likelihood of active syphilis infection). There are different possible reasons for this finding: it could be that many of the participants in our study had either been treated for a prior syphilis infection (and subsequently resulted in being serofast) or perhaps they were recently infected, resulting in their lower CD4 counts and higher VL. Interestingly, prior studies have shown that in PLWH, syphilis serological tests may not correlate with the clinical presentation, further confounding the correct diagnosis. Altered serological tests are also possible among PLWH co-infected with syphilis: post-treatment serological values may be higher than expected in serofast individuals or fluctuate; false-negative and delayed sero-reactive tests have been also described [5]. In the context of WHO guidelines for PLWH that recommend STI testing based on symptoms, these findings add to the currently limited data available on the prevalence of asymptomatic STIs among PLWH in Africa and further highlight the need for improved syphilis diagnostic testing and treatment algorithms in LIC, especially among PLWH [7]. These findings also reinforce the importance of initiating therapy with penicillin in PLWH when syphilis is diagnosed, even if not presenting with dermatological or systemic manifestations.

In the multivariate model, statistically significant risk factors for HIV and syphilis co-infection included no/ some primary education (compared to secondary education and above) and alcohol consumption. Lower educational status as a risk factor for HIV and confirmed syphilis is consistent with prior studies where Mutagoma et al. also found five times higher rates of no/primary education in PLWH and confirmed syphilis compared to those without syphilis, demonstrating the broad public health consequences of education [13]. Additionally, ART administration with HIV-1 viral suppression was found to be protective in the unadjusted model (but not adjusted model) and these results may suggest that similar factors contribute to syphilis acquisition and poor HIV virologic outcomes.

This study has several strengths to include that it evaluates a large, well-characterized cohort with a robust population of PLWH across four African countries who sought care in a variety of public and private facilities. It leverages serologic syphilis clinical data, including 
screening and confirmatory testing, which is uncommonly recorded in resource-limited settings. This study also identifies several important risk factors for PLWH and serologically-confirmed syphilis co-infection in non-pregnant individuals through multivariate analysis using demographic, clinical and socio-behavioral characteristics.

There are some limitations to this study. Our findings may not be generalized to individuals in other African regions. There is also limited AFRICOS enrollment of individuals who engage in high-risk sexual activities, such as commercial sex workers and men who have sex with men for whom there is both a higher prevalence of HIV and STI co-infection as well as a distinct characterization of socio-behavioral risk factors. In this analysis, sexual behavioral risk factors (to include accessibility to free condoms, number of lifetime sexual/casual partners, sex in exchange for gifts, money, shelter, food, drugs, and favors) were examined in an earlier bivariate model but not found to be significant and thus not included in later analytic models. Given the high proportion of serofast cases, associated risk factors may have been remote to study participation, impacting our analysis' sensitivity to detect behavior impacts on syphilis acquisition. Specifically regarding the analysis of serofast cases, our study also did not examine the correlation between AFRICOS participants' treatment history and their serological syphilis tests, limiting the ability to fully interpret these findings. Finally, our analysis did not analyze HIV and syphilis co-infection rates over time and additional studies should examine this association longitudinally so as to better guide recommendations on comprehensive care for PLWH in Africa, especially in resource-limited settings.

\section{Conclusions}

Our findings show that syphilis rates in sub-Saharan Africa remain elevated and that both lower education level and alcohol consumption are significantly associated with $\mathrm{HIV} /$ syphilis coinfection in AFRICOS. Our results also demonstrate the diagnostic challenges of syphilis infection-and suggest a role for serologic confirmatory testing, as almost half of PLWH initially diagnosed with syphilis would have received unnecessary treatment based on results of the initial (non-treponemal) screening syphilis test alone. Based on our analysis, current STI guidelines that target testing for individuals with either new HIV diagnosis or syndromic symptoms in Africa may be inadequate and highlight the need for increased testing and treatment strategies in resourcelimited settings.

\section{Abbreviations}

AFRICOS: African Cohort Study; ART: Antiretroviral therapy; HIV: Human immunodeficiency virus; HIV-1 VL: HIV-1 viral load; IHDS: International HIV Dementia Scale; IQR: Interquartile range; LIC: Low-income country; MHRP: US Military HIV Research Program; PEPFAR: President's Emergency Plan for AIDS Relief; PLWH: People living with HIV; POC: Point of care; RPR: Rapid plasma regain; STI: Sexually transmitted infection; TPA: Treponema pallidum Antibody; WHO: World Health Organization.

\section{Acknowledgements}

We thank the study participants, local implementing partners, and hospital leadership at Kayunga District Hospital, Kericho District Hospital, AC Litein Mission Hospital, Kapkatet District Hospital, Tenwek Mission Hospital, Kapsabet District Hospital, Nandi Hills District Hospital, Kisumu West District Hospital, Mbeya Zonal Referral Hospital, Mbeya Regional Referral Hospital, Defence Headquarters Medical Center, and the 68th Nigerian Army Reference Hospital.

We would also like to thank the AFRICOS Study Team members who assisted in this research study - Danielle Bartolanzo, Alexus Reynolds, Katherine Song, Mark Milazzo, Leilani Francisco, Shauna Mankiewicz, Steven Schech, Alexandra Golway, Badryah Omar, Tsedal Mebrahtu, Elizabeth Lee, Kimberly Bohince, Ajay Parikh, Jaclyn Hern, Emma Duff, Kara Lombardi, Michelle Imbach, Leigh Anne Eller, Hannah Kibuuka, Michael Semwogerere, Prossy Naluyima, Godfrey Zziwa, Allan Tindikahwa, Hilda Mutebe, Cate Kafeero, Enos Baghendaghe, William Lwebuge, Freddie Ssentogo, Hellen Birungi, Josephine Tegamanyi, Paul Wangiri, Christine Nabanoba, Phiona Namulondo, Richard Tumusiime, Ezra Musingye, Christina Nanteza, Joseph Wandege, Michael Waiswa, Evelyn Najjuma, Olive Maggaga, Isaac Kato Kenoly, and Barbara Mukanza, Jonah Maswai, Rither Langat, Aaron Ngeno, Lucy Korir, Raphael Langat, Francis Opiyo, Alex Kasembeli, Christopher Ochieng, Japhet Towett, Jane Kimetto, Brighton Omondi, Mary Leelgo, Michael Obonyo, Linner Rotich, Enock Tonui, Ella Chelangat, Joan Kapkiai, Salome Wangare, Zeddy Bett Kesi, Janet Ngeno, Edwin Langat, Kennedy Labosso, Joshua Rotich, Leonard Cheruiyot, Enock Changwony, Mike Bii, Ezekiel Chumba, Susan Ontango, Danson Gitonga, Samuel Kiprotich, Bornes Ngtech, Grace Engoke, Irene Metet, Alice Airo, and Ignatius Kiptoo, John Owuoth, Valentine Sing'oei, Winne Rehema, Solomon Otieno, Celine Ogari, Elkanah Modi, Oscar Adimo, Charles Okwaro, Christine Lando, Margaret Onyango, Iddah Aoko, Kennedy Obambo, Joseph Meyo, and George Suja, Iroezindu, Yakubu Adamu, Nnamdi Azuakola, Mfreke Asuquo, Abdulwasiu Bolaji Tiamiyu, Afoke Kokogho, Samirah Sani Mohammed, Ifeanyi Okoye, Sunday Odeyemi, Aminu Suleiman, Lawrence Umejo, Onome Enas, Miriam Mbachu, Ijeoma Chigbu-Ukaegbu, Wilson Adai, Felicia Anayochukwu Odo, Rabi Abdu, Rosemary Akiga, Helen Nwandu, CHisara Okolo, Ndubuisis Okeke, Zahra Parker, Asogwa Ugochukwu Linus, Concilia Amaka Agbaim, Tunde Adegbite, Nkenchiere Harrison, Adewale Adelakun, Ekeocha Chioma, Victoria Idi, Rachel Eluwa, Jumoke Nwalozie, Igiri Faith, Blessing Okanigbuan, Achugwo Emmanuel, Nkiru Nnadi, Ndubuisi Rosemary, Uzoegwu Amaka Natalie, Obende Theresa Owanza, Falaju Idowu Francis, Jacintal Elemere, Obilor Ifeoma Lauretta, Edward Akinwale, and Inalegwu Ochai, Lucas Maganga, Emmanuel Bahemana, Samoel Khamadi, John Njegite, Connie Lueer, Abisai Kisinda, Jaquiline Mwamwaja, Faraja Mbwayu, Gloria David, Mtasi Mwaipopo, Reginald Gervas, Doroth Mkondoo, Nancy Somi, Paschal Kiliba, Gwamaka Mwaisanga, Johnisius Msigwa, Hawa Mfumbulwa, Peter Edwin, Willyhelmina Olomi.

\section{Authors' contributions}

LG analyzed and interpreted patient data and was the primary author for the manuscript. ND and AE assisted with statistical design and data analysis. $\mathrm{MI}, \mathrm{EB}, \mathrm{HK}, \mathrm{JO}$, and JM oversaw data collection as study coordinators at their respective African sites. CP, TC, and JA assisted with study design and data analysis, where JA served as the senior research investigator for this study. All authors read and approved the final manuscript.

\section{Funding}

This work was supported by the President's Emergency Plan for AIDS Relief via a cooperative agreement between the Henry M. Jackson Foundation for the Advancement of Military Medicine, Inc., and the U.S. Department of Defense [W81XWH-11-2-0174, W81XWH-18-2-0040]. The funding body assisted with study design, data collection, analysis and interpretation of study results. 


\section{Availability of data and materials}

The datasets used and analyzed during the current study are available from the corresponding author on reasonable request.

\section{Declarations}

Ethics approval and consent to participate

All participants provided written informed consent. The study was approved by institutional review boards of the WRAIR, Makerere University School of Public Health, Kenya Medical Research Institute, Tanzania National Institute of Medical Research, and the Nigerian Ministry of Defense. The investigators have adhered to the policies for protection of human subjects as prescribed in Army Regulation 70-25. The WRAIR IRB number for this study is IRB00000794.

\section{Consent for publication}

Not applicable.

\section{Competing interests}

The authors declare that they have no competing interests. The views expressed are those of the authors and should not be construed to represent the positions of the U.S. Army or the Department of Defense or the Department of Health and Human Services. LG: I am a military service member. This work was prepared as part of my official duties. Title 17 U.S.C. 105 provides that "Copyright protection under this title is not available for any work of the United States Government."Title 17 U.S.C. 101 defines a United States Government work as a work prepared by a military service member or employee of the United States Government as part of that person's official duties.

\section{Author details}

${ }^{1}$ U.S. Military HIV Research Program, Walter Reed Army Institute of Research, Silver Spring, MD, USA. ${ }^{2}$ Henry M. Jackson Foundation for the Advancement of Military Medicine Inc., Bethesda, MD, USA. ${ }^{3} \mathrm{HJF}$ Medical Research International, Abuja, Nigeria. ${ }^{4}$ HJF Medical Research International, Mbeya, Tanzania. ${ }^{5}$ Makerere University-Walter Reed Project, Kampala, Uganda. ${ }^{6}$ U.S. Army Medical Research Directorate - Africa, Kisumu, Kenya. ${ }^{7} \mathrm{HJF}$ Medical Research International, Kisumu, Kenya. ${ }^{8} \mathrm{HJF}$ Medical Research International, Kericho, Kenya.

Received: 21 June 2021 Accepted: 30 August 2021

Published online: 30 October 2021

\section{References}

1. Newman L, Rowley J, Hoorn VS, et al. Global estimates of the prevalence and incidence of four curable sexually transmitted infections in 2012 based on systematic review and global reporting. PLoS Negl Trop Dis. 2015. https://doi.org/10.1371/journal.pone.0143304.

2. WHO: Global health sector strategy on sexually transmitted infections 2016-2021 towards ending STIs. https://www.who.int/reproductivehea Ith/publications/rtis/ghss-stis/en/; 2016.

3. Kojima N, Klausner J. An update on the global epidemiology of syphilis. Curr Epidemiol Rep. 2018;5(1):24-38.

4. Kalichman SC, Pellowski J, Turner C. Prevalence of sexually transmitted coinfections in people living with HIV/AIDS: systematic review with implications for using HIV treatments for prevention. Sex Transm Infect. 2011;87(3):183-90.

5. Magri F, Donà MG, Panetta C, Pontone M, Pimpinella F, Cameli N, Cristaudo A, Zaccarelli M, Latini A. Unusual clinical manifestation and challenging serological interpretation of syphilis: insights from a case report. BMC Infect Dis. 2021;21(1):521.

6. Kaida A, Dietrich J, Laher F, et al. A high burden of asymptomatic genital tract infections undermines the syndromic management approach among adolescents and young adults in South Africa: implications for HIV prevention efforts. BMC Infect Dis. 2018;18:499.

7. WHO: essential prevention and care interventions for adults and adolescents living with HIV in resource-limited settings. https://www.who.int/ hiv/pub/guidelines/EP/en/; 2008.

8. WHO: guidelines for the treatment of Treponema pallidum (syphilis). https://www.who.int/reproductivehealth/publications/rtis/syphilis-treat ment-guidelines/en/; 2016. Accessed 25 March 2021.

9. Ake JA, Polyak CS, Crowell TA, et al. Noninfectious comorbidity in the African Cohort Study. Clin Infect Dis. 2019:69:639-47.

10. Milanini B, Allen I, Paul R, et al. Frequency and predictors of HIV-related cognitive impairment in East Africa: The Africa Cohort Study (AFRICOS). J Acquir Immune Defic Syndr. 2020;83:157-64.

11. Djomand G, Schlefer M, Gutreuter S, et al. Prevalence and correlates of genital infections among newly diagnosed human immunodeficiency virus-infected adults entering human immunodeficiency virus care in Windhoek Namibia. Sex Transm Dis. 2016;43(11):698-705.

12. Singa B, Glick S, Bock N, et al. Sexually transmitted infections among HIVinfected adults in HIV care programs in Kenya: a national sample of HIV clinics. Sex Transm dis. 2013;40(2):148-53.

13. Mutagoma M, Remera E, Sebuhoro D, et al. Prevalence of syphilis infection and its associated factors in the general population of Rwanda: a national household-based survey. Sex Transm Dis. 2016. https://doi.org/ 10.1155/2016/4980417.

\section{Publisher's Note}

Springer Nature remains neutral with regard to jurisdictional claims in published maps and institutional affiliations.
Ready to submit your research? Choose BMC and benefit from:

- fast, convenient online submission

- thorough peer review by experienced researchers in your field

- rapid publication on acceptance

- support for research data, including large and complex data types

- gold Open Access which fosters wider collaboration and increased citations

- maximum visibility for your research: over $100 \mathrm{M}$ website views per year

At BMC, research is always in progress.

Learn more biomedcentral.com/submissions 